\title{
Effect of Prenatal Exposure to Isoflavones on Bone Metabolism in Mice at Adulthood
}

\author{
WENDY E. WARD AND ANA V. PIEKARZ \\ Department of Nutritional Sciences, Faculty of Medicine, University of Toronto, Toronto, Ontario, Canada, M5S 3E2
}

\begin{abstract}
Early postnatal exposure to genistein resulted in improved bone health at early adulthood in mice. The objective of the present study was to determine whether in utero exposure to isoflavones also has a positive effect on bone health, resulting in higher bone mineral density (BMD) and greater resistance to fracture at adulthood. Pregnant mice received daily subcutaneous injections of genistein $(3.75 \mathrm{mg})$, daidzein $(3.75 \mathrm{mg})$, genistein $(3.75 \mathrm{mg})+$ daidzein $(3.75 \mathrm{mg})$, or vehicle from d 9 to 21 of pregnancy. At birth, offspring ( $n=12 /$ group/gender) remained with their respective mother and were weaned at postnatal age of $21 \mathrm{~d}$ and fed control diet, devoid of isoflavones, until 4 mo of age, at which time tissues were collected. There was an overall effect of treatment on femur BMD, which was higher $(p<0.001)$ among control and genistein groups compared with daidzein and genistein + daidzein groups. Treatment did not have a significant effect on femur peak load. Among females, daidzein resulted in a lower ( $p=0.02$ ) BMD of lumbar vertebra (LV) 1-4 than all other groups however peak load of LV4 did not differ due to treatment or gender. In conclusion, in utero exposure to isoflavones did not result in functional benefits to bone at young adulthood. (Pediatr Res 61: 438-443, 2007)
\end{abstract}

$\mathrm{C}^{\mathrm{o}}$ onsumption of soy-based foods has steadily increased due to greater awareness of potential health benefits. Research into the ability of soy isoflavones to modulate bone metabolism has arisen largely as a result of the structural similarities between $17-\beta$-estradiol and the soy isoflavones genistein and daidzein. Because osteoporosis is often considered a disease of aging, it is perhaps not surprising that the majority of human and animal studies related to soy and/or its isoflavones have focused on preventing loss of bone mass and deterioration of bone tissue in postmenopausal women or using ovariectomized rodent models (1).

Estrogen has a critical role in skeletal development in females and males throughout life, however, the effects of isoflavone exposure during early life on bone development have not been extensively studied. In mice, manipulation of maternal estrogen levels results in alterations in the skeleton of female offspring (2). Injecting pregnant mice with synthetic estrogen, DES, during pregnancy resulted in positive, long-

Received September 27, 2006; accepted November 24, 2006.

Correspondence: Wendy E. Ward, Ph.D., Department of Nutritional Sciences, Faculty of Medicine, University of Toronto, 150 College St., FitzGerald Building, Room 316, Toronto, Ontario, Canada, M5S 3E2; e-mail: wendy.ward@utoronto.ca

W.W. holds a Future Leader Award from the International Life Sciences Institute North America (ILSI N.A.), and this work was supported, in part, by a grant from the ILSI N.A. The opinions expressed herein are those of the authors and do not necessarily represent the views of ILSI N.A.

DOI: $10.1203 /$ pdr.0b013e3180332d67 lasting effects on skeletons of female offspring, providing evidence that in utero exposure to estrogen can result in programming of bone cells (2). Whether maternal administration of isoflavones to mice has a similar effect on the skeleton of offspring is unknown. It is known that isoflavones such as daidzein are transferred from mothers to fetus (3), thereby potentially modulating biologic effects on hormone-sensitive tissues such as bone. Moreover, previous investigation demonstrated that administration of genistein to female mice during the first $5 \mathrm{~d}$ of postnatal life result in long-lasting effects on bone metabolism that were manifested at young adulthood (4). These mice that were administered genistein, at a dose similar to the level of an infant fed soy-based formula, had higher femur and spine BMD compared with controls, and the higher spine BMD resulted in stronger lumbar vertebrae that were more resistant to fracture (4).

The long-term effect of maternal exposure to isoflavones on bone development in offspring has not been studied in humans or using animal models. Human infants can be exposed to elevated levels of isoflavones in utero if their mother is consuming a diet abundant in soy foods. The objective of this study was to use a mouse model to determine whether in utero exposure to isoflavones resulted in higher BMC, BMD, and greater biomechanical bone strength properties in male and female offspring. To determine whether specific isoflavones have different effects on bone, genistein and daidzein were administered alone and in combination. The combination was studied to mimic the approximately equal distribution of genistein and daidzein in soy protein. Both femurs and lumbar vertebrae were studied to provide two specific skeletal sites, which differ in the quantity of cortical and trabecular bone. Because of potential hormonal effects of isoflavones, sex organ weights were measured at 4 mo of age.

\section{MATERIALS AND METHODS}

Animals and diets. Female and male CD-1 mice (Charles River Canada, St. Constant, QC, Canada) were adapted to control diet devoid of phytoestrogens including genistein [Dyets Inc., Bethlehem, PA, AIN93G (5)] for a period of $2 \mathrm{wk}$ and subsequently mated. Day 0 of pregnancy was determined as the day that a plug was observed. At this time, the female was housed separately and randomized to one of the following study groups: control $(0.4$ $\mathrm{mL}$ corn oil), genistein ( $3.75 \mathrm{mg}$ genistein in $0.4 \mathrm{~mL}$ corn oil), daidzein ( 3.75 $\mathrm{mg}$ daidzein in $0.4 \mathrm{~mL}$ corn oil), or genistein + daidzein (3.75 mg genistein $+3.75 \mathrm{mg}$ daidzein in $0.4 \mathrm{~mL}$ corn oil) from d 9 of pregnancy until d 21 of pregnancy. Isoflavones were administered as subcutaneous injections. Doses

Abbreviations: BMC, bone mineral content; BMD, bone mineral density; DES, diethylstilbestrol 
of isoflavones were based on the level of isoflavones in a soy protein-based rodent diet in which approximately $500 \mathrm{mg}$ of isoflavones are present per kilogram of diet. Based on previous data indicating that pregnant mice consume approximately $10 \mathrm{~g}$ of diet per day, and after adjustment for the fact that isoflavones were administered by subcutaneous injection (reduced dose by $25 \%$ ), a dose of $3.75 \mathrm{mg}$ was determined appropriate. From d 9 through $\mathrm{d}$ 21 of pregnancy, mothers were injected once daily with their respective treatment. At birth, pups remained with their mother until they were weaned at postnatal age of $21 \mathrm{~d}$ and housed four per cage. For each treatment group there were 24 pups ( 12 females +12 males). Throughout the study, mice were kept in standard environment conditions (12-h light:12-h dark cycle, room temperature of $23^{\circ} \mathrm{C}$ ), and fresh diet was provided every $2-3 \mathrm{~d}$. Distilled water was consumed ad libitum. Body weight was measured once weekly between 0700 and $0800 \mathrm{~h}$. Total weight gain was calculated by subtracting body weight at randomization (PND 21) from body weight at necropsy (PND 120). At 4 mo of age, mice were anesthetized with carbon dioxide for blood collection, and subsequently killed by carbon dioxide overdose. Femurs and LV1-LV4 were excised and stored at $-70^{\circ} \mathrm{C}$. Testes (males), seminal vesicle (males), prostate (males), uterus (females), and ovaries (females) were removed and weighed to monitor for gross changes due to potential hormonal activity of isoflavones.

All experimental procedures respected the policies set out by the Canadian Council on Animal Care (6) and were approved by the University of Toronto Animal Ethics Committee, University of Toronto.

Biochemical markers of bone metabolism. Blood was centrifuged at $10,000 \mathrm{rpm}$ for $15 \mathrm{~min}$ and serum was stored at $-70^{\circ} \mathrm{C}$. Serum osteocalcin and serum collagen crosslinks were measured using commercially available ELISA specific for mice (Mouse osteocalcin, Biotechnologies Inc., Stoughton, MA; RatLaps, Nordic Bioscience Diagnostics, Herlev, Denmark). Intraassay and inter-assay coefficient of variation were $7.7 \%$ and $6.3 \%$ for osteocalcin, and $7.6 \%$ and $10.1 \%$ for the collagen crosslinks assay.

$B M C$ and BMD of femurs and lumbar vertebrae (LV1-LV4). BMC and BMD of the left femurs and LV1-LV4 were determined by PIXImus dual energy x-ray absorptiometry (DEXA) (LUNAR Corporation, GE Medical Systems, Mississauga, ON, Canada) using a specialized software program (Lunar Software Version 1.46) as previously described (7). Individual femurs and intact lumbar vertebrae (LV1-LV4) were placed on a plastic tray and scanned in air.

Biomechanical strength properties of femurs and lumbar vertebra 3 (LV3). The biomechanical strength properties of the femur and LV4 were measured by three-point bending and compression testing, respectively, using a material testing system (Model 4442, Instron Corp., Canton, MA) and specialized software (Series IX Automated Materials Tester, Version 8.15.00).

Three-point bending. Three-point bending was used to determine the structural properties of right femurs $(7,8)$. Femurs previously cleaned of soft tissue were removed from the freezer and soaked in physiologic saline solution for $3 \mathrm{~h}$. Length and widths of femurs were measured using an electronic precision caliper (Cedarlane Laboratories Ltd., Hornby, ON, Canada). The femur midpoint was determined based on the length of the femur. The depth (anteroposterior width) and width (mediolateral width) dimensions were measured at the midpoint of the femur. The posterior surface of the femur was placed on two 1-mm-wide base supports that were positioned 6 $\mathrm{mm}$ apart. The bones were positioned on the supports such that the load was applied at the midpoint of the bone shaft. The crosshead lowered at a constant speed of $2 \mathrm{~mm} / \mathrm{min}$ until the femur fractured. The biomechanical properties that were determined were yield point (a measure of the elastic limit of a bone), peak load (a measure of the maximum force that the bone withstood before fracture), and stiffness (a measure of the extrinsic rigidity of the bone tissue). Yield load primarily measures the contribution of mineral to bone strength. In contrast, peak load is a measurement of the contribution of bone matrix proteins to the strength of the bone.

Compression testing. Peak load of LV4 was determined as previously described $(7,8)$. LV4 were rehydrated and positioned on the center of a stainless-steel plate. The height, depth (anteroposterior width), and width (mediolateral width) of each LV4 were measured using an electronic precision caliper. A compressive force was applied by lowering a steel plate at a constant rate of $2 \mathrm{~mm} / \mathrm{min}$. Peak load was determined to be the first peak on the load-displacement curve.

Statistical analysis. Statistical analyses were performed using SigmaStat (Version 2.0, Jandel Scientific, San Rafael, CA). Results are expressed as mean \pm SEM. Two-way ANOVA was performed for all outcomes, except sex organ weights. Gender and treatment were tested as main effects and testing for interactions (gender $\times$ treatment) was assessed. One-way ANOVA was used to analyze weights of sex organs.

For both one-way and two-way ANOVA, Student-Newman Keul's test was used for comparison of multiple means when statistical differences were observed. To determine the relationship between whole femur BMC and biomechanical strength properties (yield load, stiffness, peak load), linear regression analyses were performed. For all analyses, differences were considered significant at $p<0.05$.

\section{RESULTS}

Pregnancy outcomes. There were no differences in pregnancy outcomes including number of pups per litter (number of pups/litter: control $=11 \pm 1$; genistein $=11 \pm 1$; daidzein $=11 \pm 2$; genistein + daidzein $=12 \pm 2$ ); number of males and females per litter (f, m: control $=6,5$ genistein $=$ 6,5 daidzein $=7,4$; genistein + daidzein $=4,7$ ); and birth weight (in grams, control $=1.99 \pm 0.24$; genistein $=1.94 \pm$ 0.14 ; daidzein $=1.99 \pm 0.03$; genistein + daidzein $=1.92 \pm$ 0.17 ) among treatment groups.

Weaning weight, final body weight and total weight gain. Weaning weight of control and genistein alone groups were significantly greater $(p=0.011)$ than the genistein + daidzein group (Table 1). Final body weight $(p<0.001)$ and total weight gain $(p<0.001)$ was significantly higher among males compared with females with no differences due to treatment (Table 1).

Table 1. Body weight and total weight gain*

\begin{tabular}{|c|c|c|c|c|c|}
\hline Treatment & Males & Females & Gender & Treatment & Gender $\times$ treatment \\
\hline \multicolumn{6}{|l|}{ Weaning weight $(\mathrm{g}) \dagger$} \\
\hline Control & $13.5 \pm 1.1$ & $12.0 \pm 0.9$ & NS & 0.011 & NS \\
\hline Genistein & $12.7 \pm 0.8$ & $12.4 \pm 0.4$ & & & \\
\hline Genistein + daidzein & $10.5 \pm 0.7$ & $10.5 \pm 0.6$ & & & \\
\hline \multicolumn{6}{|l|}{ Final body weight $(\mathrm{g}) \ddagger$} \\
\hline Control & $46.1 \pm 2.2$ & $32.7 \pm 1.4$ & $<0.001$ & NS & NS \\
\hline Genistein + daidzein & $44.4 \pm 2.1$ & $32.4 \pm 1.0$ & & & \\
\hline \multicolumn{6}{|l|}{ Total weight gain (g) } \\
\hline Control & $32.5 \pm 1.5$ & $20.7 \pm 1.3$ & $<0.001$ & NS & NS \\
\hline Genistein & $30.8 \pm 1.6$ & $19.8 \pm 1.8$ & & & \\
\hline Daidzein & $31.2 \pm 1.1$ & $22.0 \pm 0.8$ & & & \\
\hline Genistein + daidzein & $33.9 \pm 1.8$ & $22.0 \pm 0.8$ & & & \\
\hline
\end{tabular}

* Values are expressed as mean \pm SEM.

$\dagger$ Body weight at weaning, postnatal d 21 .

$\ddagger$ Body weight at necropsy, postnatal d 120 . 
Organ weights. The weights of testes, prostate, and seminal vesicle expressed as absolute weight (not shown) or corrected to account for body weight were not different among groups (Table 2). Similarly, weights of uterus and ovaries, expressed either as absolute weight (not shown) or corrected to account for body weight, were not different among the three groups (Table 2).

Femur outcomes: weight, dimensions, $B M C, B M D$ and biomechanical strength properties. Femur weight was higher ( $p=0.022)$ among males compared with females and there was a treatment effect with the daidzein group having higher $(p=0.037)$ femur weight than the genistein + daidzein group (Table 3). Length and mediolateral width of femurs did not differ among groups due to gender or treatment (Table 3). Anteroposterior width of femurs was higher among males compared with females, and daidzein group also had greater anteroposterior width than all other groups (Table 3).

Femur BMC did not differ among groups due to gender or treatment (Table 4$)$ but BMD was higher $(p<0.001)$ among control and genistein groups compared with daidzein alone and genistein + daidzein group (Table 4$)$.

Femur yield load was greater $(p=0.035)$ among males compared with females (Table 4$)$. There was a significant ( $p=0.007$ ) interaction between gender and treatment. Females receiving genistein had higher $(p=0.007)$ yield load than female controls and genistein treated males (Table 4). Control males had a higher $(p=0.007)$ yield load than female control group (Table 4). Femur peak load did not differ due to treatment but males had a higher $(p<0.001)$ peak load than females (Table 4). For ultimate stiffness, there was an overall effect due to treatment but not gender (Table 4). Genistein and daidzein groups had higher $(p=0.038)$ ultimate stiffness than control group (Table 4).

Dimensions of lumbar vertebrae 4 (LV4), BMC, and BMD of LV1-LV4, and Peak Load of LV4. Height of LV4 was greater $(p=0.035)$ in the genistein group compared with the daidzein group, with no differences due to gender (Table 5). Weight of LV4 was higher ( $p=0.037)$ among the control group compared with the genistein + daidzein group (Table 5). Depth did not differ among groups due to gender or treatment (Table 5) but width of control group was greater $(p=0.023)$ than all other groups (Table 5).

BMC of LV1-LV4 did not differ due to gender but control group had a higher $(p=0.03)$ BMC of LV1-LV4 compared with daidzein and genistein + daidzein group (Table 6). A higher BMD of LV1-LV4 ( $p=0.003$ ) was observed in the control and genistein group compared with the daidzein alone group (Table 6). There was a significant interaction between gender and treatment, with females treated with daidzein having a lower $(p=0.02)$ BMD of LV1-LV4 than control

Table 2. Sex organ weights*

\begin{tabular}{|c|c|c|c|c|c|}
\hline Treatment & $\begin{array}{c}\text { Testes } \\
\text { (mg/g body } \\
\text { weight) }\end{array}$ & $\begin{array}{c}\text { Prostate } \\
\text { (mg/g body } \\
\text { weight) }\end{array}$ & $\begin{array}{c}\text { Seminal vesicle } \\
\text { (mg/g body } \\
\text { weight })\end{array}$ & $\begin{array}{c}\text { Uterus } \\
\text { (mg/g body } \\
\text { weight) }\end{array}$ & $\begin{array}{c}\text { Ovaries } \\
\text { (mg/g body } \\
\text { weight) }\end{array}$ \\
\hline Control & $4.61 \pm 0.33$ & $1.22 \pm 0.11$ & $7.14 \pm 0.47$ & $2.91 \pm 0.39$ & $0.42 \pm 0.05$ \\
\hline Genistein & $5.61 \pm 0.50$ & $0.97 \pm 0.27$ & $7.62 \pm 0.47$ & $3.19 \pm 0.37$ & $0.59 \pm 0.03$ \\
\hline Daidzein & $5.16 \pm 0.21$ & $1.04 \pm 0.10$ & $5.47 \pm 0.45$ & $2.98 \pm 0.32$ & $0.65 \pm 0.07$ \\
\hline Genistein + daidzein & $5.36 \pm 0.37$ & $1.12 \pm 0.12$ & $6.37 \pm 0.49$ & $3.10 \pm 0.40$ & $0.57 \pm 0.11$ \\
\hline
\end{tabular}

* Values are expressed as mean \pm SEM.

Table 3. Femur weight and dimensions*

\begin{tabular}{|c|c|c|c|c|c|}
\hline Treatment & Males & Females & Gender & Treatment & Gender $\times$ treatment \\
\hline \multicolumn{6}{|l|}{ Weight (mg) } \\
\hline Control & $87.50 \pm 6.29$ & $80.83 \pm 6.56$ & 0.022 & 0.037 & NS \\
\hline Genistein & $85.83 \pm 2.87$ & $73.33 \pm 1.88$ & & & \\
\hline Daidzein & $89.09 \pm 4.75$ & $87.50 \pm 4.62$ & & & \\
\hline Genistein + daidzein & $80.00 \pm 3.48$ & $68.33 \pm 4.05$ & & & \\
\hline \multicolumn{6}{|l|}{ Length (mm) } \\
\hline Control & $16.62 \pm 0.11$ & $16.55 \pm 0.10$ & NS & NS & NS \\
\hline Genistein & $16.38 \pm 0.10$ & $16.14 \pm 0.12$ & & & \\
\hline Daidzein & $16.47 \pm 0.16$ & $16.54 \pm 0.11$ & & & \\
\hline Genistein + daidzein & $16.39 \pm 0.15$ & $16.25 \pm 0.09$ & & & \\
\hline \multicolumn{6}{|l|}{ Depth $\dagger(\mathrm{mm})$} \\
\hline Control & $1.45 \pm 0.02$ & $1.40 \pm 0.02$ & NS & NS & NS \\
\hline Genistein & $1.49 \pm 0.04$ & $1.47 \pm 0.04$ & & & \\
\hline Daidzein & $1.47 \pm 0.03$ & $1.44 \pm 0.03$ & & & \\
\hline Genistein + daidzein & $1.41 \pm 0.02$ & $1.40 \pm 0.02$ & & & \\
\hline \multicolumn{6}{|l|}{ Width $末$ (mm) } \\
\hline Control & $1.91 \pm 0.03$ & $1.71 \pm 0.03$ & $<0.001$ & $<0.001$ & NS \\
\hline Genistein & $1.85 \pm 0.04$ & $1.71 \pm 0.03$ & & & \\
\hline Daidzein & $2.11 \pm 0.07$ & $1.85 \pm 0.06$ & & & \\
\hline Genistein + daidzein & $1.86 \pm 0.02$ & $1.63 \pm 0.04$ & & & \\
\hline
\end{tabular}

* Values are expressed as mean \pm SEM.

$\uparrow$ Depth refers to the anteroposterior width at the midpoint of the femur.

$\ddagger$ Width refers to the mediolateral width at the midpoint of the femur. 
Table 4. Femur BMC, BMD, and biomechanical strength properties*

\begin{tabular}{|c|c|c|c|c|c|}
\hline Treatment & Males & Females & Gender & Treatment & Gender $\times$ treatment \\
\hline \multicolumn{6}{|l|}{ BMC (mg) } \\
\hline Control & $40.51 \pm 0.99$ & $36.42 \pm 0.73$ & NS & NS & NS \\
\hline Genistein & $40.00 \pm 1.00$ & $36.83 \pm 1.23$ & & & \\
\hline Genistein + daidzein & $37.09 \pm 1.27$ & $38.3 \pm 4.34$ & & & \\
\hline \multicolumn{6}{|l|}{$\operatorname{BMD}\left(\mathrm{mg} / \mathrm{cm}^{2}\right)$} \\
\hline Control & $76.43 \pm 0.88$ & $74.35 \pm 1.37$ & NS & $<0.001$ & NS \\
\hline Genistein + daidzein & $72.80 \pm 1.15$ & $67.42 \pm 3.10$ & & & \\
\hline \multicolumn{6}{|l|}{ Yield load $(\mathrm{N})$} \\
\hline Control & $16.30 \pm 1.09^{\mathrm{ab}}$ & $14.0 \pm 0.53^{\mathrm{c}}$ & NS & 0.035 & 0.007 \\
\hline Genistein & $14.67 \pm 0.74^{\mathrm{bc}}$ & $17.38 \pm 0.46^{\mathrm{a}}$ & & & \\
\hline Daidzein & $17.05 \pm 0.92$ & $16.40 \pm 0.61$ & & & \\
\hline Genistein + daidzein & $14.33 \pm 0.64$ & $15.02 \pm 0.58$ & & & \\
\hline Genistein + daidzein & $28.60 \pm 1.05$ & $26.26 \pm 0.51$ & & & \\
\hline \multicolumn{6}{|l|}{ Ultimate stiffness $(\mathrm{N} / \mathrm{mm})$} \\
\hline Control & $206.44 \pm 8.63$ & $183.91 \pm 4.56$ & NS & 0.038 & NS \\
\hline Genistein & $197.65 \pm 8.38$ & $217.30 \pm 7.30$ & & & \\
\hline Daidzein & $209.17 \pm 10.91$ & $218.67 \pm 7.88$ & & & \\
\hline Genistein + daidzein & $191.05 \pm 7.41$ & $195.41 \pm 6.29$ & & & \\
\hline
\end{tabular}

* Values are expressed as mean \pm SEM

Values with different letters for an outcome are significantly different, $p<0.05$.

Table 5. Dimensions of Lumbar Vertebra 4 (LV4)*

\begin{tabular}{|c|c|c|c|c|c|}
\hline Treatment & Males & Females & Gender & Treatment & Gender $\times$ treatment \\
\hline \multicolumn{6}{|l|}{ Height (mm) } \\
\hline Control & $3.75 \pm 0.11$ & $3.51 \pm 0.09$ & NS & 0.035 & NS \\
\hline Genistein & $3.96 \pm 0.11$ & $3.82 \pm 0.16$ & & & \\
\hline Genistein + daidzein & $3.63 \pm 0.11$ & $3.69 \pm 0.10$ & & & \\
\hline \multicolumn{6}{|l|}{ Weight (mg) } \\
\hline Control & $47.27 \pm 5.20$ & $41.66 \pm 5.40$ & NS & 0.037 & NS \\
\hline Genistein + daidzein & $29.09 \pm 3.92$ & $36.36 \pm 3.8$ & & & \\
\hline \multicolumn{6}{|l|}{$\operatorname{Depth} \dagger(\mathrm{mm})$} \\
\hline Control & $3.17 \pm 0.08$ & $3.11 \pm 0.07$ & NS & NS & NS \\
\hline Genistein & $3.01 \pm 0.05$ & $3.10 \pm 0.06$ & & & \\
\hline Daidzein & $3.14 \pm 0.07$ & $3.18 \pm 0.03$ & & & \\
\hline Genistein + daidzein & $3.17 \pm 0.05$ & $2.98 \pm 0.04$ & & & \\
\hline Genistein + daidzein & $3.29 \pm 0.06$ & $3.23 \pm 0.04$ & & & \\
\hline
\end{tabular}

* Values are expressed as mean \pm SEM

$\dagger$ Depth refers to the anteroposterior width of LV4.

$\ddagger$ Width refers to the mediolateral width of LV4.

females and genistein-treated females (Table 6). Peak load of LV4 did not differ due to treatment or gender (Table 6).

Biochemical markers: serum osteocalcin and serum collagen crosslinks. Serum osteocalcin was higher $(p<0.001)$ among females than males, and no treatment effect was observed (Table 7). Serum collagen crosslinks did not differ due to gender or treatment (Table 7).

Relationship of whole femur BMC to biomechanical bone strength properties. Whole femur BMC was positively and significantly related to yield load ( $r=0.274, p=0.009)$, ultimate stiffness $(r=0.271, p=0.010)$ and peak load $(r=$ 0.462, $p<0.001$ ) at the femur midpoint (Fig. 1).

\section{DISCUSSION}

This study has shown that prenatal exposure to genistein, daidzein, or the combination of genistein + daidzein does not result in greater BMD or biomechanical bone strength than 
Table 6. BMC and BMD of lumbar vertebrae (LV1-LV4) and peak load of LV4*

\begin{tabular}{|c|c|c|c|c|c|}
\hline Treatment & Males & Females & Gender & Treatment & Gender $\times$ treatment \\
\hline \multicolumn{6}{|l|}{ BMC (mg) } \\
\hline Control & $33.84 \pm 0.99$ & $31.72 \pm 1.14$ & NS & 0.03 & NS \\
\hline Genistein & $28.80 \pm 0.72$ & $31.30 \pm 1.52$ & & & \\
\hline Genistein + daidzein & $31.00 \pm 1.05$ & $29.71 \pm 0.71$ & & & \\
\hline \multicolumn{6}{|l|}{$\operatorname{BMD}\left(\mathrm{mg} / \mathrm{cm}^{2}\right)$} \\
\hline Control & $74.29 \pm 1.36^{\mathrm{ab}}$ & $72.20 \pm 1.44^{\mathrm{a}}$ & NS & 0.003 & 0.02 \\
\hline Genistein + daidzein & $71.01 \pm 1.11^{\mathrm{ab}}$ & $70.70 \pm 0.86^{\mathrm{ab}}$ & & & \\
\hline \multicolumn{6}{|l|}{ Peak load $(\mathrm{N})$} \\
\hline Control & $53.31 \pm 3.79$ & $44.04 \pm 4.18$ & NS & NS & NS \\
\hline Genistein & $46.14 \pm 2.73$ & $55.60 \pm 3.78$ & & & \\
\hline Daidzein & $38.95 \pm 3.54$ & $47.34 \pm 4.01$ & & & \\
\hline Genistein + daidzein & $45.94 \pm 4.11$ & $38.25 \pm 4.69$ & & & \\
\hline
\end{tabular}

* Values are expressed as mean \pm SEM.

Values with different letters for an outcome are significantly different, $p<0.05$.

Table 7. Biochemical markers of bone metabolism: serum osteocalcin and serum collagen crosslinks*

\begin{tabular}{|c|c|c|c|c|c|}
\hline Treatment & Males & Females & Gender & Treatment & Gender $\times$ treatment \\
\hline Control & $106.71 \pm 9.16$ & $154.8 \pm 14.49$ & $<0.001$ & NS & NS \\
\hline Genistein & $117.00 \pm 23.65$ & $164.00 \pm 12.38$ & & & \\
\hline Genistein + daidzein & $106.71 \pm 9.16$ & $150.30 \pm 14.68$ & & & \\
\hline \multicolumn{6}{|c|}{ Collagen crosslinks (ng/mL) } \\
\hline Control & $40.50 \pm 2.01$ & $37.97 \pm 5.73$ & NS & NS & NS \\
\hline Genistein + daidzein & $41.85 \pm 3.23$ & $40.80 \pm 2.23$ & & & \\
\hline
\end{tabular}

* Values are expressed as mean \pm SEM.

offspring not treated with isoflavones. However, of interest is the observation that genistein and daidzein had differing effects on femur and lumbar spine BMD. While femur BMD of genistein-treated mice did not differ significantly from control, mice treated with daidzein or the combination of gensitein + daidzein had lower femur BMD than both control and genistein groups. This finding suggests that when the isoflavones are provided in combination, as occurs in soy foods, the effect of daidzein alone persists in the presence of genistein. Similarly, exposure to daidzein alone resulted in a lower BMD of lumbar spine compared with control and genistein groups but did not differ from mice exposed to the combination of genistein + daidzein. The differences in femur and lumbar spine BMD did not result in differences in peak load at femur midpoint or LV4 indicating that genistein and daidzein do not have contrasting effects with respect to bone strength.
Whether or not the lower BMD persists and may have effects on bone strength and thus risk of fracture at later stages of life is uncertain. Functional differences in bone, i.e. bone strength, were not observed due to exposure to isoflavones, but regression analyses revealed that femur BMC was a statistically significant predictor of bone strength properties in these mice. However, the correlation coefficients were low $(0.271,0.274$, and 0.462$)$, and suggest that small changes in BMD are not directly translated into weaker or stronger bones. A recent paper that investigated whether changes in BMD were related to changes in fracture risk concluded that BMD measurements reflect only one component in bone strength (9) and that interventions can have functional effects independent of dramatic changes in BMD.

In this study, bone outcomes were studied at 4 mo of age, a time at which peak bone mass is achieved in mice (10). Bio-
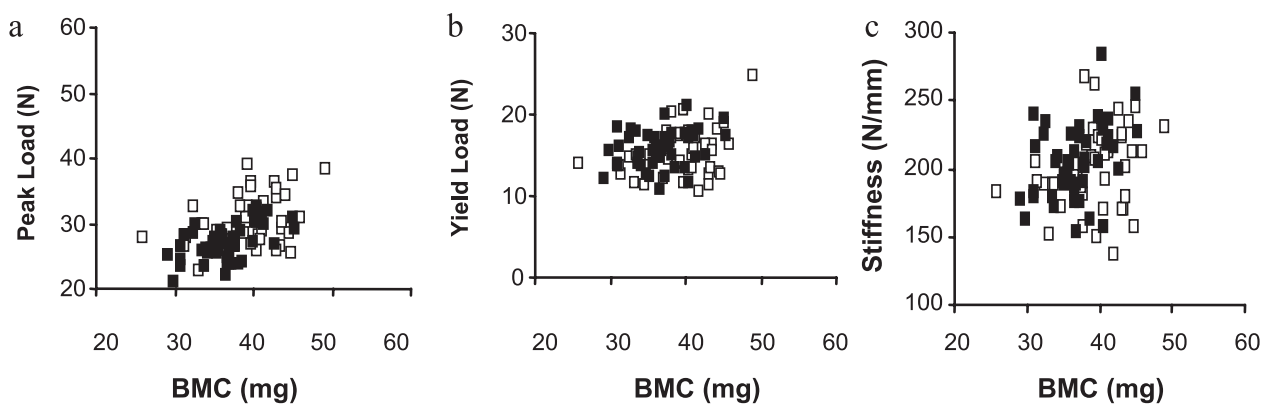

Figure 1. Relationship of whole femur BMC and (a) peak load $(r=0.462, p<$ $0.001)$; $(b)$ yield load $(r=0.274, p=0.009)$; and $(c)$ stiffness $(r=0.271, p=0.010)$ at the femur midpoint. Solid squares represent females; open squares represent males. 
chemical markers of bone turnover were not different due to treatment, suggesting that differences in both the rates of bone formation and bone resorption that would have led to differences in femur and lumbar spine BMD occurred before this age. Determining at what age BMD is altered should be determined to more fully understand how the ontogeny of bone mineral accumulation is altered with prenatal exposure to isoflavones. Serial measurements of BMD and biochemical markers of bone turnover would elucidate the timing of these changes and potential differences among treatments at specific stages of development.

It is known that the breed of mice used in this study, CD-1 mice, can efficiently convert daidzein to equol (11). It is purported that equol may have greater estrogenic activity than daidzein (12), and thus it is possible that equol may be mediating the effect of daidzein on femur and lumbar spine BMD. Conversion of daidzein to equol occurs in the large intestine; equol produced in the mother's intestine is passed across the placenta (13). Moreover, genistein and daidzein are also transferred from mother to fetus (13). Serum levels of isoflavones were assessed at time of C-section delivery from the mother and infant (cord blood) to assess the potential level of fetal exposure to maternal isoflavones (13). Of potential biologic importance was the finding that isoflavones have a longer life in the fetus than in the mother, providing a basis for isoflavones to modulate effects in the fetus (13). A similar transfer from mother to fetus has been reported in rats; daidzein is rapidly transferred from the maternal circulation to the fetus (3) and elevated levels of genistein, daidzein, and equol are observed in rat fetuses at gestational $\mathrm{d}$ 16.5 and 20.5 from mothers fed isoflavones (14). Moreover, newborn rat pups born to mothers consuming soy-containing diets have high circulating levels of isoflavones (15).

The overall lack of effect of isoflavones on bone outcomes is in contrast to the changes on fetal bone development observed after alteration of estrogen levels of mothers with administration of synthetic estrogen, DES, during pregnancy (2). In utero exposure to DES modulates skeletal development rapidly, as 16-d-old fetuses had greater skeletal calcification than untreated fetuses (2). Moreover, when these mice were kept to young adulthood, they had higher mineral apposition rate. The fact that circulating estrogen levels at young adulthood were unchanged compared with untreated controls suggest that the changes in bone metabolism were due to cell programming, and not mediated by hormonal changes. When comparing these study findings to the present study it is important to recall that the dose of isoflavone administered was chosen, to the best of our ability, to reflect a level of isoflavone exposure that a human infant might receive in utero. The dose of synthetic estrogen (DES) administered in the aforementioned study had significantly greater estrogenicity. It is estimated that DES is approximately 50,000 times more estrogenic than an equivalent dose of genistein (16). It is also possible that the timing of exposure during pregnancy is a factor influencing the effect of isoflavones on bone metabolism. In this study, pregnant mice were exposed to isoflavones at d 9 of pregnancy as previous investigators reported that exposure to synthetic estrogen (DES) at this stage of pregnancy resulted in long-term programming of bone tissue. It is possible that earlier intervention with isoflavones may have modulated bone metabolism.
Sex organ weights did not differ at 4 mo of age; however, to be certain of long-term potential toxicological effects of in utero exposure to isoflavones, histologic analyses of organs from mice at older ages, i.e. $>12$ mo of age, should be assessed as it is known that toxicological effects of environmental estrogens in mice may not be observed until later in life. Previous investigation in male mice suggests that exposure to dietary levels of genistein in utero through the end of suckling does not alter reproductive indices, including sperm quality (i.e. fertilizing ability) and testicular gene expression (17). Another study in male and female offspring exposed to dietary levels of genistein, in utero, also report no negative effects on reproductive indices such as eyelid opening, puberty onset, and anogenital distance among other measures (18).

In conclusion, in utero exposure to individual isoflavones, genistein or daidzein, or their combination did not confer a positive, long-lasting effect on bone health as BMD and biomechanical strength properties were not greater than control group at 4 mo of age, representing young adulthood. Based on the findings that genistein and daidzein may have differing effects on bone health, future investigations should explore the mechanisms behind these different responses. With respect to human studies, long-term follow-up studies are needed to determine whether consumption of soy foods during pregnancy modulates bone health of the child later in life.

Acknowledgments. The authors thank A. Eryou for assistance with animal care and collection of tissues.

\section{REFERENCES}

1. Reinwald S, Weaver CM 2006 Soy isoflavones and bone health: a double-edged sword? J Nat Prod 69:450-459

2. Migliaccio S, Newbold RR, Bullock BC, Jefferson WJ, Sutton FG Jr, McLachlan JA, Korach KS 1996 Alterations of maternal estrogen levels during gestation affect the skeleton of female offspring. Endocrinology 137:2118-2125

3. Degen GH, Janning P, Diel P, Michna H, Bolt HM 2002 Transplacental transfer of the phytoestrogen daidzein in DA/Han rats. Arch Toxicol 76:23-29

4. Piekarz A, Ward WE 2007 Effect of neonatal exposure to genistein on bone metabolism in mice at adulthood. Pediatr Res 61:48-53

5. Reeves PG 1997 Components of the AIN-93 diets as improvements in the AIN-76A diet. J Nutr 127:838S-841S

6. Canadian Council on Animal Care 1984 Guide to the Care and Use of Experimental Animals. Ottawa, ON, Canada

7. Fonseca D, Ward WE 2004 Daidzein together with high calcium preserve bone mass and biomechanical strength at multiple sites in ovariectomized mice. Bone 35:489-497

8. Turner CH, Burr DB 2001 Experimental techniques for bone mechanics. In: Cowin SC (ed) Bone Mechanics Handbook. CRC Press, Boca Raton, FL, pp 7.1-7.34

9. Rubin CD 2005 Emerging concepts in osteoporosis and bone strength. Curr Med Res Opin 218:1049-1056

10. Beamer WG, Donahue LR, Rosen CJ, Baylnk DJ 1996 Genetic variability in adult bone density among inbred strains of mice. Bone 18:397-403

11. Ward WE, Kim S, Chan D, Fonseca D 2005 Serum equol, bone mineral density and biomechanical bone strength differ among four mouse strains. J Nutr Biochem 16:743-749

12. Setchell KD, Brown NM, Lydeking-Olsen E 2002 The clinical importance of the metabolite equol-a clue to the effectiveness of soy and its isoflavones. J Nutr 132:3577-3578

13. Todaka E, Sakurai K, Fukata H, Miyagawa H, Uzuki M, Omori M, Osada H, Ikezuki Y, Tsutsumi O, Iguchi T, Mori C 2005 Fetal exposure to phytoestrogens-the difference in phytoestrogen status between mother and fetus. Environ Res 99:195-203

14. Weber KS, Setchell KD, Lephart ED 2001 Maternal and perinatal brain aromatase: effects of dietary soy phytoestrogens. Brain Res Dev Brain Res 126:217-221

15. Brown NM, Setchell KD 2001 Animal models impacted by phytoestrogens in commercial chow: implications for pathways influenced by hormones. Lab Invest 81:735-747

16. Jefferson WN, Newbold RR 2000 Potential endocrine-modulating effects of various phytoestrogens in the diet. Nutrition 16:658-662

17. Fielden MR, Samy SM, Chou KC, Zacharewski TR 2003 Effect of human dietary exposure levels of genistein during gestation and lactation on long-term reproductive development and sperm quality in mice. Food Chem Toxicol 41:447-454

18. Kang KS, Che JH, Lee YS 2002 Lack of adverse effects in F1 offspring maternally exposed to genistein at human intake dose level. Food Chem Toxicol 40:43-51 